\title{
Imaging Protein-Protein Interactions By Multiphoton FLIM
}

\author{
S. M. Ameer-Beg ${ }^{1}$, N. Edme ${ }^{2}$, M. Peter ${ }^{2}$, P. R. Barber ${ }^{1}$, T. $\mathrm{Ng}^{2}$, B. Vojnovic ${ }^{1}$. \\ 1. Advanced Technology Development Group, Gray Cancer Institute, Mount Vernon Hospital, \\ Northwood, Middlesex, HA6 2JR, UK. \\ 2. Randall Centre, Guys Medical School Campus, Kings College London, SE1 1UL, UK.
}

\begin{abstract}
We demonstrate the applicability of time-correlated single photon counting multiphoton microscopy to the spatiotemporal localisation of protein-protein interactions in situ. An Example of a new fluorescent protein variant with enhanced properties are given and the development of a FRET biosensor for simultaneous measurement of multiple intra- and inter-molecular interactions is illustrated by experimental evidence of an energy transfer cascade via multiple acceptors. The juxtaposition of interacting population and FRET efficiency is elucidated, with a priori knowledge, by multi-exponential analysis.
\end{abstract}

Keywords: multiphoton imaging, TCSPC, FRET, FLIM, fluorescent proteins, GFP.

\section{INTRODUCTION}

The spatio-temporal localisation of protein-protein interactions within cells in situ and in particular in vivo is of great importance in elucidating the key mechanisms in regulation of the fundamental process of the cell. Measurements of such near-field localisation of protein complexes may be achieved by the detection of fluorescence (or Förster) resonance energy transfer (FRET) between protein-conjugated fluorophores ${ }^{1}$. The dependence of the coupling efficiency varies with the inverse-sixth power of the distance between acceptor and donor and is typically described in terms of the Förster radius (distance at which the efficiency of energy transfer is 50\%), usually of the order 1-10 nm. The process depletes the excited state population of the donor such that FRET will both reduce the fluorescence intensity and the fluorescence lifetime of the donor. The advantage of using donor fluorescence lifetime imaging microscopy (FLIM) to detect FRET, as opposed to intensity-based measurements, arises due to the independence of fluorophore concentration and light path length and is therefore well suited to studies in intact cells ${ }^{2,3}$. Combined with confocal or multiphoton microscopical techniques to examine the localisation of effects in cellular compartments, FLIM/FRET techniques allow us to determine populations of interacting protein species on a point-by-point basis at each resolved voxel in the cell ${ }^{4}$.

Analysis of FRET between interacting proteins (i.e. intermolecular FRET) is often hampered by the dichotomy of interacting fraction of donor-accepter pairs and FRET efficiency. In a simple case, where all donors are bound to an acceptor, the donor fluorescence lifetime will have a single exponential lifetime modified by the distance between donor and acceptor fluorophores. However, in a mixed population, where there is a distribution of molecular separations or unbound donor fluorophores, multiple exponential decay kinetics are likely to be observed. The presence of noninteracting species has a negative effect on the determination of FRET efficiency. High FRET efficiency but low concentration of interacting molecules may then lead to the incorrect assumption that there is little or no interaction between FRET partners. Localisation of protein interaction to compartments below the imaging resolution leads to an averaging of the effect over the measured volume and thereby potential misinterpretation of the biological effects. Given appropriate data, analysis of the spatial distributions of fluorophores may be determined.

We present examples of FRET experiments for proteins implicated in motility and metastasis using a number of different biological protocols to elucidate mechanisms of protein interaction in the signalling cascade. We discuss the use of a new fluorescent protein pair for FRET and underline the merits and disadvantages for specific applications. In addition, we present a new FRET technique to enable the simultaneous measurement of protein conformation and intramolecular binding via an apparent excited state saturation of an acceptor. We use a donor-acceptor(donor)-acceptor regime which enables us to examine the conformation of a protein (in the example presented, p21 activated Kinase 1 (PAK 1)) in the presence of a second binding protein which initiates a conformational change (an activated mutant of cdc42 (V12 cdc42)). Evidence of a cascaded FRET through the protein construct is presented for both wide field spectral imaging and multiphoton FLIM. 


\section{MATERIALS AND METHODS}

\section{TIME-DOMAIN MULTIPHOTON FLUORESCENCE LIFETIME IMAGING}

Time-domain FLIM was performed with a multiphoton microscopy system, based on a modified Bio-Rad MRC 1024MP workstation, as described previously ${ }^{5}$. Enhanced non-descanned detection was afforded by the use of in-house developed detectors, situated in the re-projected pupil plane. Fluorescence lifetime imaging capability was incorporated, with addition of time-correlated single photon counting (TCSPC) electronics (Becker \& Hickl, SPC 700). The instrument response was measured from the hyper-Rayleigh scattering of highly attenuated excitation in a suspension of $20 \mathrm{~nm}$ colloidal gold (Sigma-Aldrich Company Ltd, G-1652) mounted on a coverslip ${ }^{6}$ and found to be $\sim 170$ ps fullwidth at half maximum and used in the analysis of data by iterative re-convolution. Data was collected either at $500 \pm$ $20 \mathrm{~nm}$ (Coherent Inc. 35-5040) for eGFP emission and $600 \pm 20 \mathrm{~nm}$ (Coherent Inc. 35-5081) for either cy3 or mRFP. Laser power was adjusted to give average photon counting rates of the order $10^{4}-10^{5}$ photons $\mathrm{s}^{-1}(0.0001$ to 0.001 photons per excitation event) and with peak rates approaching $10^{6}$ photons $\mathrm{s}^{-1}$, below the maximum counting rate afforded by the TCSPC card to avoid pulse pile-up. Analysis of the fluorescence transients was performed either with the SPCimage software package (Becker \& Hickl Gmbh, Berlin) or using in-house developed analysis tools.

\section{WIDE-FIELD SPECTRAL IMAGING}

The spectral imaging device used for this study was developed and constructed in our Institute and has been described in detail elsewhere ${ }^{7}$. It uses a standard monochrome CCD camera (type 4912, Cohu Inc., USA). A spectrally selective element is placed between the camera and the microscope output port using standard C-mount couplers and is based on a linearly variable dielectric band-pass filter together with novel drive hardware and acquisition software. The element has a resolution of $15 \mathrm{~nm}$ and covers the $400 \mathrm{~nm}$ to $700 \mathrm{~nm}$ band with a transmission of $>50 \%$. For this study, the spectrally resolved device was used with an upright microscope (Optiphot, Nikon, UK) equipped with a conventional fluorescence attachment with a fluorescence cube for excitation at $480 \pm 10 \mathrm{~nm}$ and broadband $(>500 \mathrm{~nm}) \mathrm{emission}$ filter with collection between 500 and $690 \mathrm{~nm}$.

\section{CELL CULTURE AND TRANSFECTION}

All cells were maintained in Dulbecco's Modified Eagle's Medium supplemented with 10\% fetal calf serum. For FRET/FLIM analysis, MDA-MB-231 breast cancer cells were microinjected using an Eppendorf microinjection system. Post-injection, cells are returned to the incubator and allowed to quiesce or express the protein of interest for 2-3 h, these were fixed in $4 \%$ paraformaldehyde for $15 \mathrm{~min}$, permeabilised with $0.2 \%$ in $0.2 \%$ Triton X-100/PBS and then either viewed directly or stained with a cy3-anti-myc mAb (and cy5-anti-HA antibodies where indicated).

\section{ANALYSIS OF FLUORESCENCE LIFETIME IMAGING DATA FOR FRET}

Determination of the interacting sub-population of proteins is of crucial importance in analysis of intermolecular FRET data. The measurement data set for FRET by fluorescence lifetime measurement of the donor species, may contain both interacting and non-interacting protein populations, which are distinct in their decay kinetics. The interacting FRET pairs have a decay rate given by:

$$
k_{\text {fret }}=\frac{1}{\tau_{d}}\left(\frac{R_{0}}{r}\right)^{6}
$$

Where $\tau_{\mathrm{d}}$ is the donor lifetime in the absence of the acceptor, $R_{0}$ is the Förster radius and $r$ the separation between the fluorophores. The Förster radius for a number of fluorescent protein pairs from the literature is given in Table $1^{8}$.

\begin{tabular}{|c|c|c|c|c|c|c|}
\hline \multicolumn{2}{|c|}{} & \multicolumn{5}{|c|}{ Acceptor } \\
\cline { 3 - 7 } \multicolumn{2}{|c|}{} & CFP & eGFP & YFP & Cy3 & Cy5 \\
\hline \multirow{2}{*}{$\tilde{\circ}$} & CFP & - & $4.7^{*}$ & $4.9^{*}$ & - & - \\
\cline { 2 - 7 } & eGFP & - & $4.6^{*}$ & $5.5^{*}$ & $6.0^{*}$ & $4.0^{+}$ \\
\cline { 2 - 7 } & Cy3 & - & - & - & $4.4^{*}$ & $5.3^{*}$ \\
\hline
\end{tabular}

Table 1. Forster Radii in nanometers for relevant fluorophore pairs $\left({ }^{*}\right.$ Harpur \& Bastiaens. ${ }^{+}$Unpublished data).

The FRET efficiency is related to the molecular separation of donor and acceptor and the fluorescence lifetime of the interacting fraction by: 


$$
\eta_{\text {fret }}=\left(\frac{R_{0}^{6}}{{R_{0}}^{6}+r^{6}}\right)=1-\frac{\tau_{\text {fret }}}{\tau_{d}}
$$

Where $R_{0}$ is the Förster radius, $R$ the molecular separation, $\tau_{\text {fret }}$ is the lifetime of the interacting fraction and $\tau_{D}$ the lifetime of the donor in the absence of acceptor. This may be re-arranged to show that:

$$
r=\sqrt[6]{\frac{\left(1-\eta_{\text {fret }}\right) R_{0}{ }^{6}}{\eta_{\text {fret }}}}=\sqrt[6]{\frac{\left(\frac{\tau_{\text {fret }}}{\tau_{d}}\right) R_{0}{ }^{6}}{\left(1-\frac{\tau_{\text {fret }}}{\tau_{d}}\right)}}
$$

In Figure 1A, $\mathrm{r}$ is plotted as a function of $\tau_{\text {fret }}$, clearly illustrating that accurate determination of the molecular separation of fluorophores can only occur at, or near, the Förster radius where the variation in lifetime is relatively linear. However, determinations of molecular separation are rarely quoted in the literature, due to uncertainty in the real value of $\mathrm{R}_{0}$.

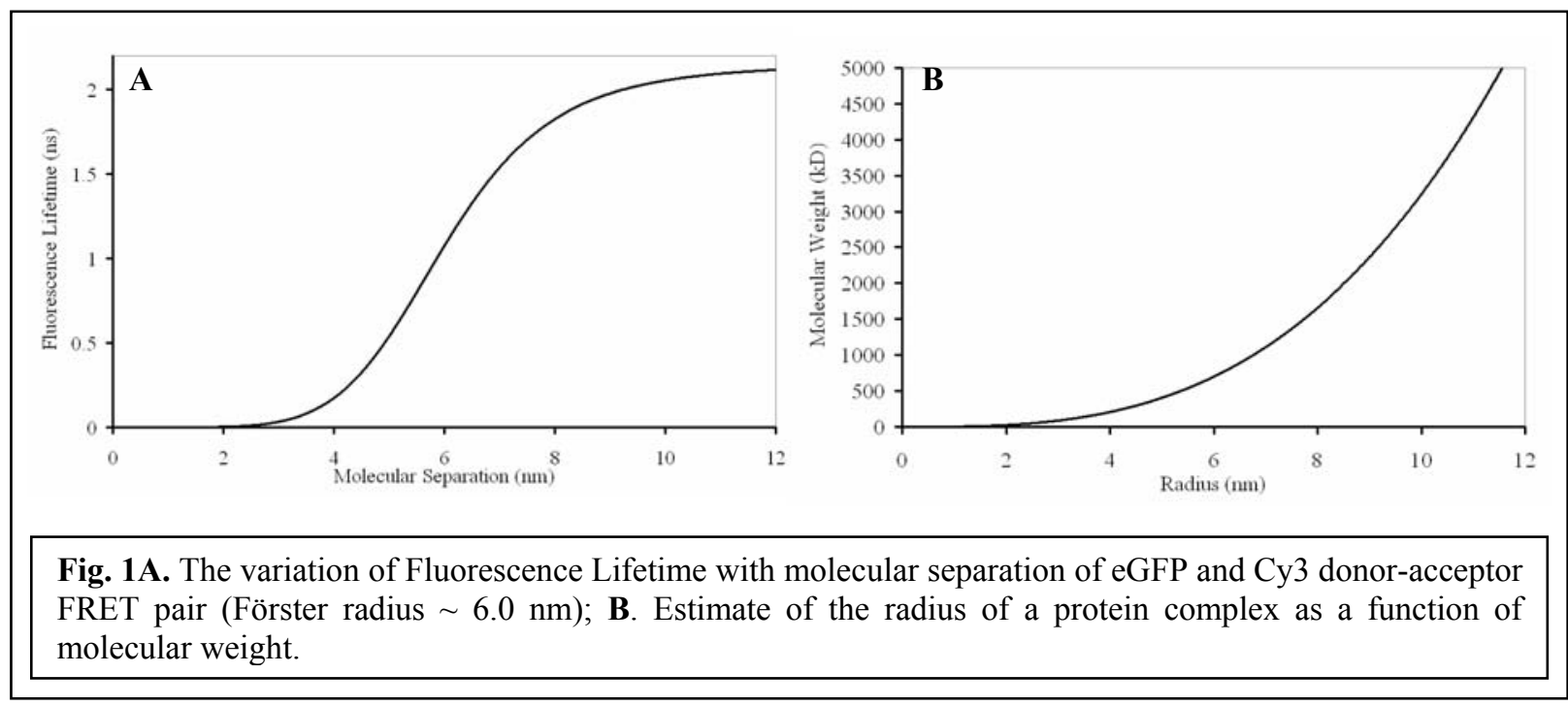

When measuring protein-protein interactions it's useful to consider the size of the molecules of interest. As a crude approximation we consider globular proteins assuming the specific volume of a protein to be $0.74 \mathrm{~cm}^{3} \mathrm{~g}^{-1}$. The radius, $\mathrm{R}$, of a globular protein of molecular weight, MW, can be estimated from the equation (fig. $1 \mathrm{~b})^{8}$ :

$$
R=\left(6.76 \times 10^{-2}\right) \quad \sqrt[3]{M W} \quad(\mathrm{~nm})
$$

Since a complex may consist of a number of sub-units, which only loosely associate, it is useful to consider both the radius of the complex in terms of a summed MW and a sum of radii for each protein in the complex to give an overview of the possible fluorophore separation range.

Since there will be a distribution of $r$ values for the ensemble of donor molecules it would be most appropriate to consider application of a complex distributed lifetime model to the experimental data and recover the distance distribution of fluorophores, as has been demonstrated by Rolinski et al. ${ }^{9}$. However, given the complexity of this approach and the need for extremely high photon counts to achieve statistically relevant results, we assume that only two populations of donor molecules are present, i.e. interacting and non-interacting populations. We apply a biexponential fluorescence decay model to the data in order to determine the fluorescence lifetime of non-interacting and interacting sub-populations. The data may be fitted by iterative re-convolution to: 


$$
I(t)=\int_{-\infty}^{\infty} I_{\text {instr }}(t)\left\{I_{0}\left(\alpha_{1} \operatorname{Exp}\left(-t / \tau_{1}\right)+\alpha_{2} \operatorname{Exp}\left(-t / \tau_{2}\right)\right)\right\} d t
$$

Where $\mathrm{I}_{\text {instr }}$ is the instrumental response, $\mathrm{I}_{0}$ the peak intensity, $\alpha_{1}$ and $\alpha_{2}$ are the fractional proportions of the lifetimes, $\tau_{1}$ and $\tau_{2}$ respectively. The reduced goodness-of-fit parameter, $\chi_{r}^{2}$, is used as defined by Lakowicz ${ }^{10}$ :

$$
\chi_{r}^{2}=\frac{\sum_{k=1}^{n} \frac{\left[I\left(t_{k}\right)-I_{c}\left(t_{k}\right)\right]^{2}}{I\left(t_{k}\right)}}{n-p}
$$

where $\mathrm{I}\left(\mathrm{t}_{\mathrm{k}}\right)$ is the data and $\mathrm{I}_{\mathrm{c}}\left(\mathrm{t}_{\mathrm{k}}\right)$ the fit value at the $\mathrm{k}^{\text {th }}$ time point, $\mathrm{t}_{\mathrm{k}} \cdot \mathrm{n}$ is the number of time points and $\mathrm{p}$ the number of variable fit parameters. $\chi_{\mathrm{r}}{ }^{2}$ is minimised using a modified Marquardt algorithm and compared alongside plots for the weighted residuals $\left(R=\left(I\left(t_{k}\right)-I_{c}\left(t_{k}\right)\right) / I\left(t_{k}\right)\right)$ to determine the validity of the decay model.

In analysis of FRET data (particularly for protein-interaction applications) there are usually two elements that must be considered, interacting fluorophore population and FRET efficiency. Bulk measurements of FRET efficiency (i.e. intensity based methods) cannot distinguish between an increase in FRET efficiency (i.e. coupling efficiency) and an increase in FRET population (concentration of FRET species), since the two parameters are not resolved. Measurements of FRET, based on analysis of the fluorescence lifetime of the donor may resolve this dichotomy when analysed using multi-exponential decay models. The assumption that non-interacting and interacting fractions are present allows us to determine both the efficiency of interaction (i.e. from $\tau_{1}$ ) and the fractional population of interacting population (i.e. $\alpha_{1}$ ). Such an approach may be extended from a pixel-by-pixel analysis (where we make no spatial restraints on the fitting parameters) to analysis of all pixels globally under the assumption that the FRET efficiency is constant across all pixels (so called 'global analysis') ${ }^{11}$. 


\section{RESULTS AND DISCUSSION NEW FLUORESCENT PROTEIN VARIANT FOR FRET}

We have investigated a number of new variant fluorescent proteins as donor-acceptor pairs for use in time-resolved multiphoton microscopy and compared their usefulness as probes for intra- and inter-molecular interactions with the

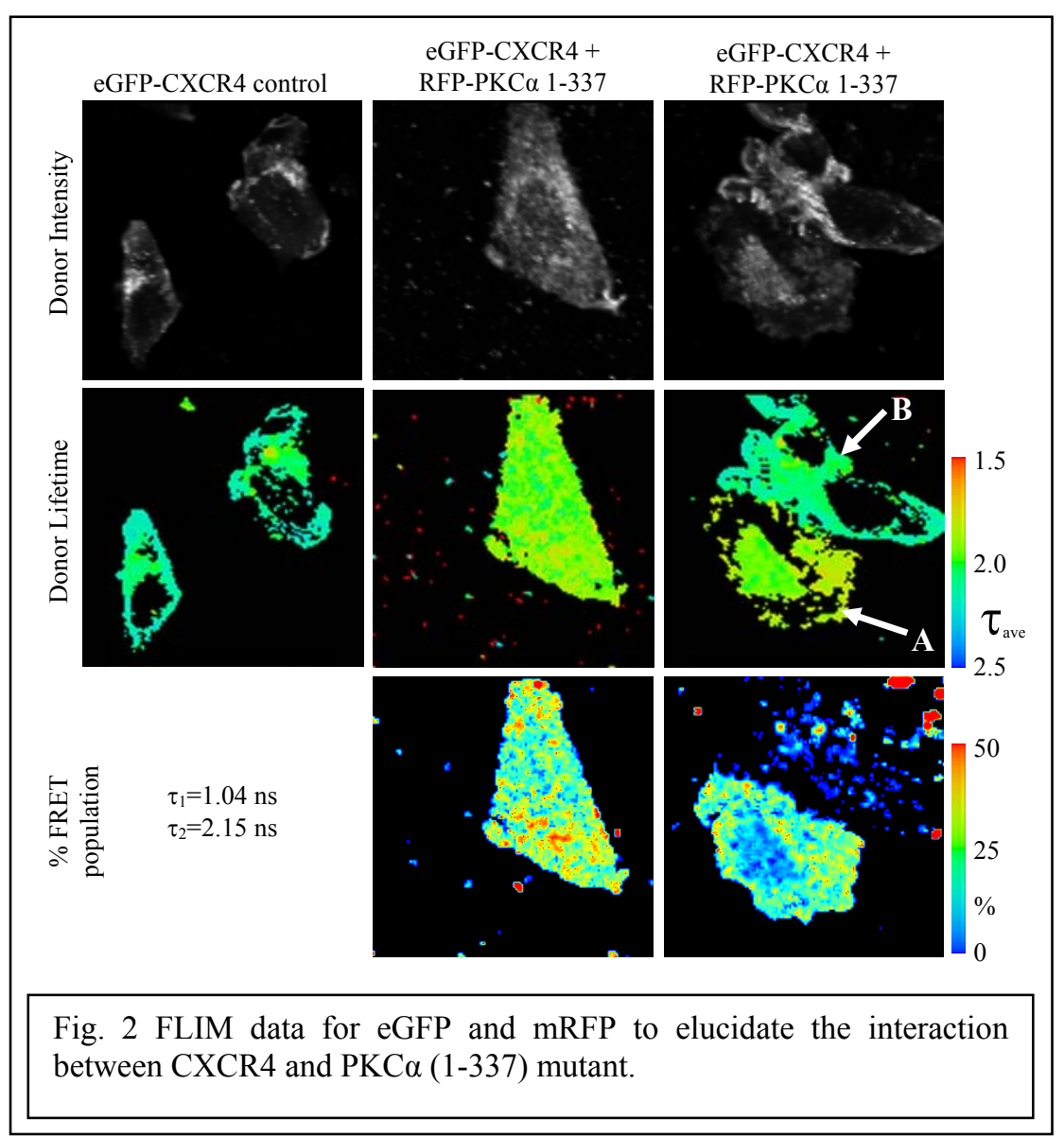
more mature techniques. A variety of different fluorescent molecules may be used as donor and acceptor pairs; the most exciting amongst these are the fluorescent protein variants due primarily to their use as dynamic probes of interaction in situ. The use of cyan and yellow fluorescent proteins (CFP and YFP) has dominated the literature ${ }^{12}$, despite a number of problems with this approach (not least the biphasic decay of CFP). We have used the new monomeric variant of red fluorescent protein $(\mathrm{mRFP})^{13}$ as an acceptor for eGFP. We have found that it is not effected by the cross-talk, characteristic of the trimeric slow maturing variant dsRed. In the example give in Fig. 2, eGFP-CXCR4 was co-transfected with an mRFPPKC $\alpha$ mutant (1-337) and found to undergo significant energy transfer. The average lifetime of the donor was observed to decrease from the control eGFP lifetime of $2.15 \mathrm{~ns}$ to $\sim 1.8 \mathrm{~ns}$ with a clearly bi-phasic decay. In order to examine the relationship between FRET efficiency and interaction population we undertake a restrained bi-exponential fit to the data. We assume the control lifetime of eGFP ( $2.15 \mathrm{~ns})$ and a fast component ( $\tau \sim 1.04 \mathrm{~ns})$, derived from an unrestrained fit to the data. This reveals the proportion of interacting species at each pixel. An average FRET efficiency of $\sim 0.51$ is thereby resolved with an typical interacting fraction of $30 \%$. A field of cells with (A) and without (B) mRFP gives a direct control for the data under identical conditions. Reduction of donor fluorescence lifetime was not observed for cells transfected with a non-interacting protein pair, indicating that the reduction in lifetime is not due to emission from the mRFP. Development of an expressible protein as acceptor for eGFP, represents a significant advantage over the CFPYFP pair for quantitative imaging, since the fluorescence decay observed for CFP is typically biphasic even in the absence of $\mathrm{YFP}^{14}$. In addition, the low absorption and poor quantum yield for CFP make it a poor choice compared to eGFP.

\section{CASCADE BIOSENSOR - CONCERTED BINDING AND CONFORMATIONAL CHANGES}

We have developed a new FRET technique, which we term a 'FRET-cascade', to examine the relationship between conformational change and binding which is equally applicable in vivo and in vitro. The principle of operation of the cascade FRET biosensor is given in Figure 3A. We use a donor-acceptor (donor)-acceptor regime, which enables us to examine the conformation of a protein in the presence of a second binding protein which may, or may not, have initiated the conformational change. The sensor is similar to that proposed by Hell and co-workers ${ }^{15,16}$, except that in that case the fluorophores in the FRET cascade were deliberately closely coupled to ensure highly efficient resonant energy transfer. For the purpose of this report, the biosensor comprises a protein construct (protein A) incorporating 
eGFP as a primary donor at the C-terminus and a myc-epitope at the N-terminus (analogous to the ubiquitous 'cameleon'-type biosensor ${ }^{17}$ ) and a second protein construct (protein B) incorporating an HA-epitope. If the constructs are expressed in cells and later stained with appropriate labelled antibodies to myc and HA, we can observe FRET between both the primary FRET pair (intra-molecular FRET) and the secondary pair (intermolecular FRET) either individually, by exciting the donor for each pair in turn and detecting the donor lifetime in each case, or collectively, by exciting only the primary donor and making measurements for both the primary donor and it's acceptor. Measurement of both FRET pairs simultaneously has the advantage that the molecular colocalisation of each interaction is determined (i.e. conformation AND binding has occurred) rather than inferring that to be the case by independent measurements.

We have employed the FRET cascade to examine the relationship between p21 activated kinase 1 (PAK 1$)^{18}$ and an activated mutant of cdc42 (V12 cdc42). PAK1 is an effecter of Rac/Cdc42 GTPases that has been implicated in the regulation of cytoskeletal dynamics, proliferation, and cell survival signaling ${ }^{19}$. In this case donor eGFP is conjugated to the N-terminus of PAK expressing a myc tag on the C-terminus. Labelling of the C-terminus with a cy3-IgG raised against myc allows in situ monitoring of protein folding of PAK, similar to common cameleon biosensors. A construct of V12 cdc42 with an HA tag is used to provide a second acceptor site for FRET from cy3-IgG and is labelled with an IgG raised against HA. This arrangement enables measurement of both conformation and binding by independent measurement of FRET between either the eGFP and IgG-myc-cy3 or IgGmyc-cy3 and IgG-HA-cy5. Excitation of eGFP and simultaneous monitoring of the eGFP and cy3 fluorescence lifetime allows the determination of correlated populations of both conformational change and binding. Nominally in a closed form (Fig 3B), PAK unfolds on binding to stimulated cdc42 (and the active mutant V12 in the absence of stimulation) and is concentrated in vesicular structures. PAK has been shown to form dimers in vitro in both inactive (closed) and active (open) forms $^{20,21}$. However, dimer formation will inevitably be strongly dependent on

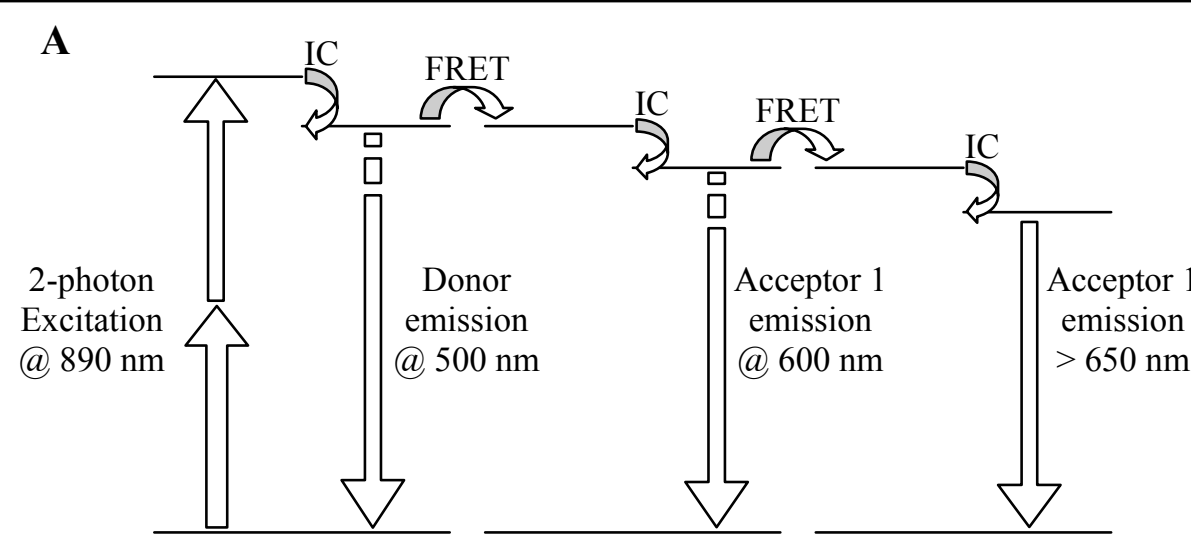

Donor 1 eGFP

Acceptor 1

Donor 2

Cy3

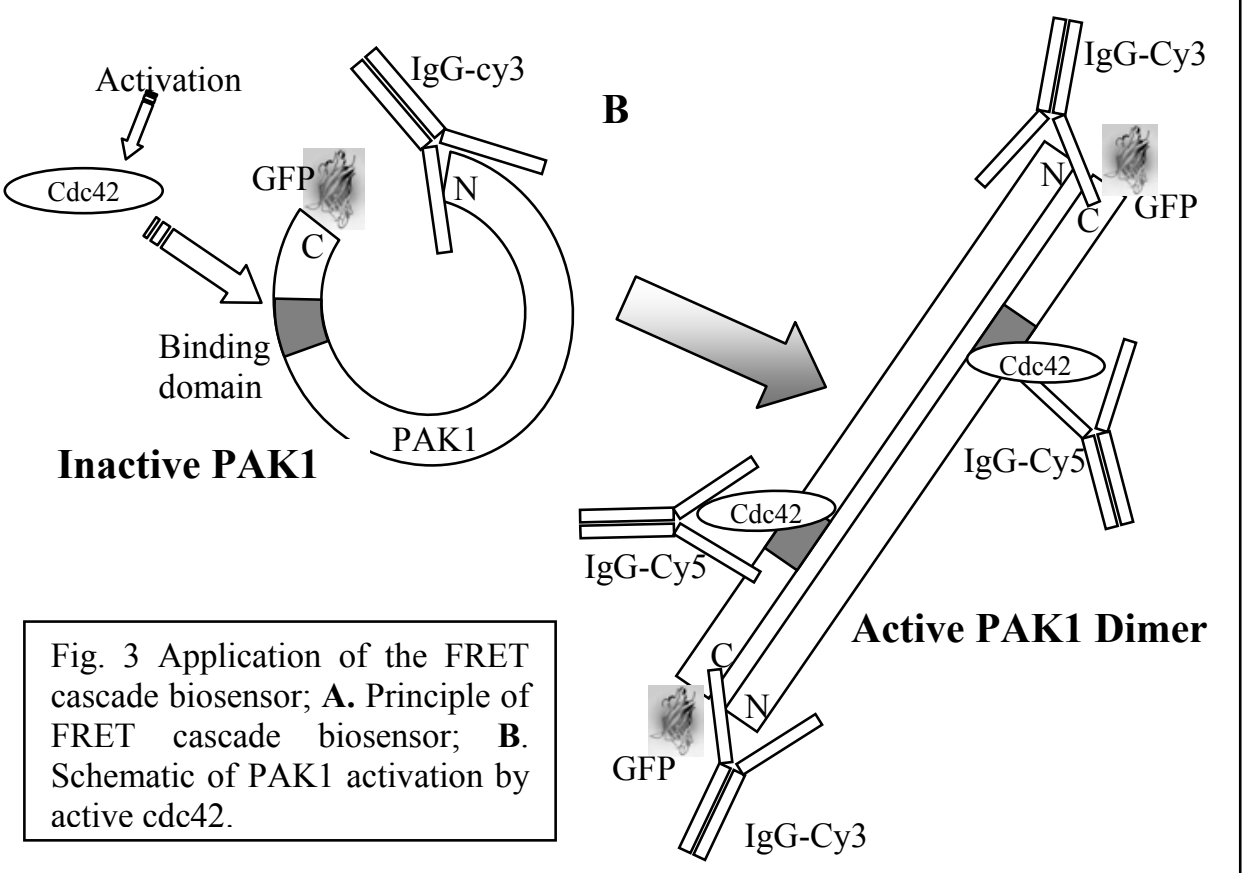
protein concentration within cells and may be organelle specific. The formation of dimers of the open active form (i.e. 
as represented in Fig. 3B) would be observed as an apparent increase in FRET efficiency by intermolecular FRET in contrast to the intramolecular FRET between C and N, which should be eliminated in the presence of V12 cdc42.

\section{QUALITATIVE DETERMINATION OF FRET CASCADE: SPECTRAL IMAGING}

Qualitative screening of samples to determine the presence of colocalisation of the three fluorescent markers was undertaken by spectral imaging (Fig. 4). Figure 4A-C shows three images of cells transfected with both eGFP-PAKmyc and V12 cdc42, corresponding to $520 \mathrm{~nm}$ (green), $580 \mathrm{~nm}$ (Red) and $650 \mathrm{~nm}$ (Blue) for eGFP, cy3 and cy5 respectively. The control (A) clearly shows structure in the peri-nuclear region which is thought to be associated with

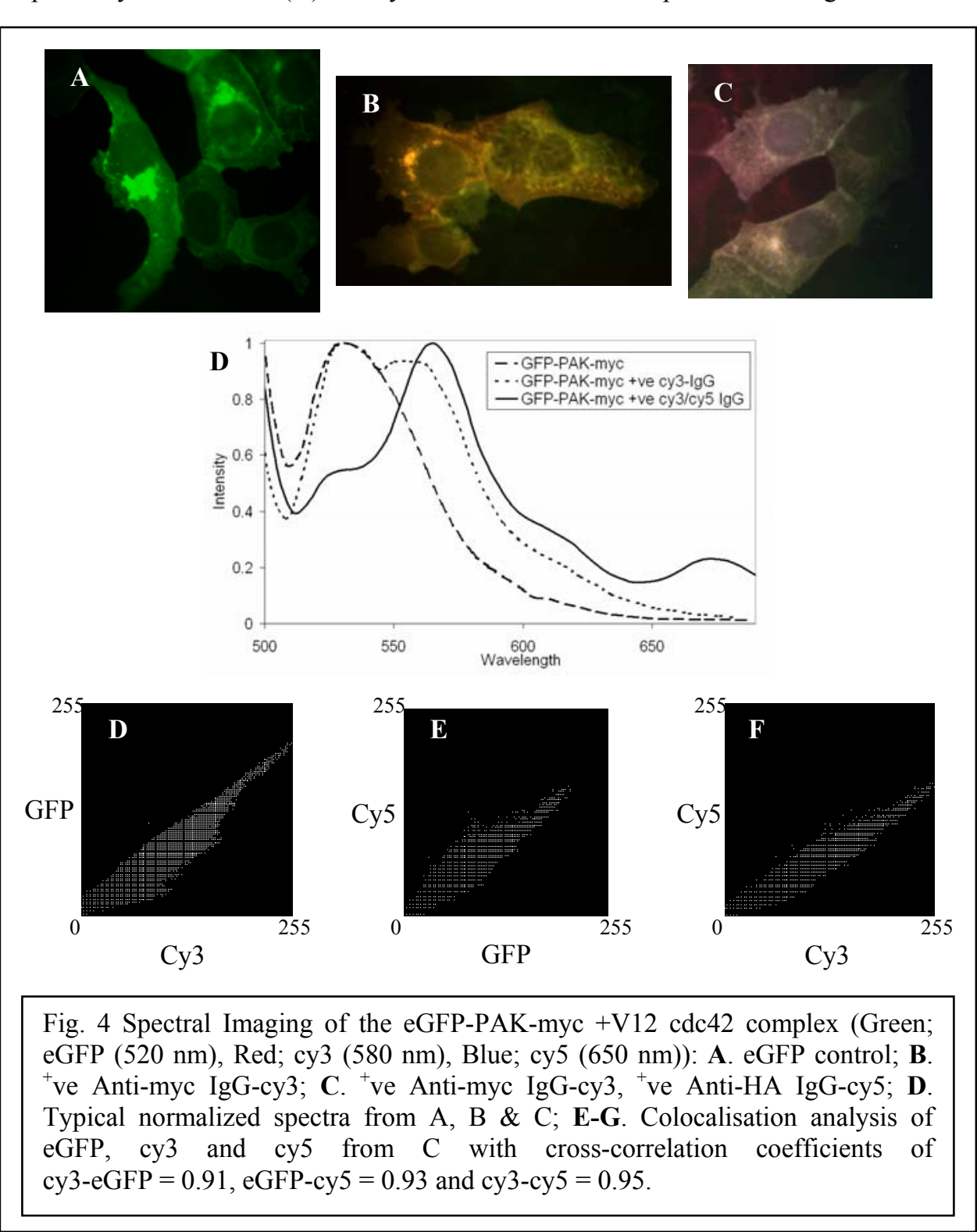

the golgi apparatus (confirmed by partial colocalisation with giantin, T. Ng data not shown). This localisation does not occur for eGFP-PAK alone, suggesting that activation (opening) of PAK by active cdc42 stimulates accumulation in this organelle. Normalised fluorescence spectra for a region of interest in each image is shown in fig.4 D. Addition of cy3 labeled anti-body to the myc epitope clearly introduces a shoulder or dual peak in the spectra compared to eGFPPAK alone, indicative of FRET between the $\mathrm{N}$ and $\mathrm{C}$ terminus of the PAK molecule. However, no correction is made for nonresonant excitation of cy 3 . In the presence of cy5 an additional peak is observed at $\sim 670 \mathrm{~nm}$ suggesting additional coupling to cy5 via cy3 and qualitative confirmation of an energy cascade from eGFP. Interestingly the cy3 peak is far more pronounced for the tri-labelled samples suggesting that the presence of a cy5 labelled antibody increases emission from cy3 relative to eGFP. Such a result is counter intuitive, since we expect energy to be transferred via cy3 to cy5 thereby reducing emission from cy3. This may simply represent an increase in anti-myc IgG-cy3 concentration in Fig.4C, relative to Fig. 4B. However, the apparent increase in coupling between eGFP and cy3 in the presence of cy5 is confirmed in the lifetime imaging results presented in the next section. Colocalisation of the three fluorophores is confirmed in fig. 4E-F with cross-correlation factors in the range $0.91-0.95$. With additional controls for non-resonant excitation of cy 3 and cy 5 in the absence of eGFP and non- 
specific anti-bodies, this spectral imaging data would be adequate to perform quantitative measurements of FRET efficiency through the cascade biosensor. In addition, the use of linear un-mixing of reference spectra would greatly improve the sensitivity of the technique without the need to correct for filter bleed-through.

\section{MULTIPHOTON FLUORESCENCE LIFETIME IMAGING OF THE CASCADE BIOSENSOR}

In order to quantitatively validate the cascade biosensor we have undertaken fluorescence lifetime imaging of the eGFP donor in the presence and absence of either or both cy3 and cy5 labelled antibodies and the results are presented in Figure 5. In control cells containing only eGFP-PAK-myc (V12 cdc42 negative) we observe a mono-exponential decay with a lifetime of the order of 2.15 ns (typical for most eGFP labelled proteins under the fixing conditions employed). In the presence of anti-myc IgG-cy3, we observed a slight reduction in fluorescence lifetime compared with the control (A), which was observed in all measurements $(\mathrm{N}=5)$. In the presence of $\mathrm{V} 12 \mathrm{cdc} 42$, we observe a marked change in the distribution of eGFP-PAK (as for the spectral imaging results in Fig. 4), with a partial colocalisation with the golgi apparatus. Typically, unfolding (in the presence of V12 cdc42) should increase the separation between donor and acceptor, recovering the donor fluorescence lifetime. However, FRET efficiency is observed to increase in the presence of anti-myc IgG-cy3, due to formation of $\mathrm{C}$ to $\mathrm{N}$ dimers of PAK bound to V12 cdc42. This has been confirmed by biochemical methods (T.Ng, data not shown). In the presence of cy5-IgGHA we observe an anomalous increase in
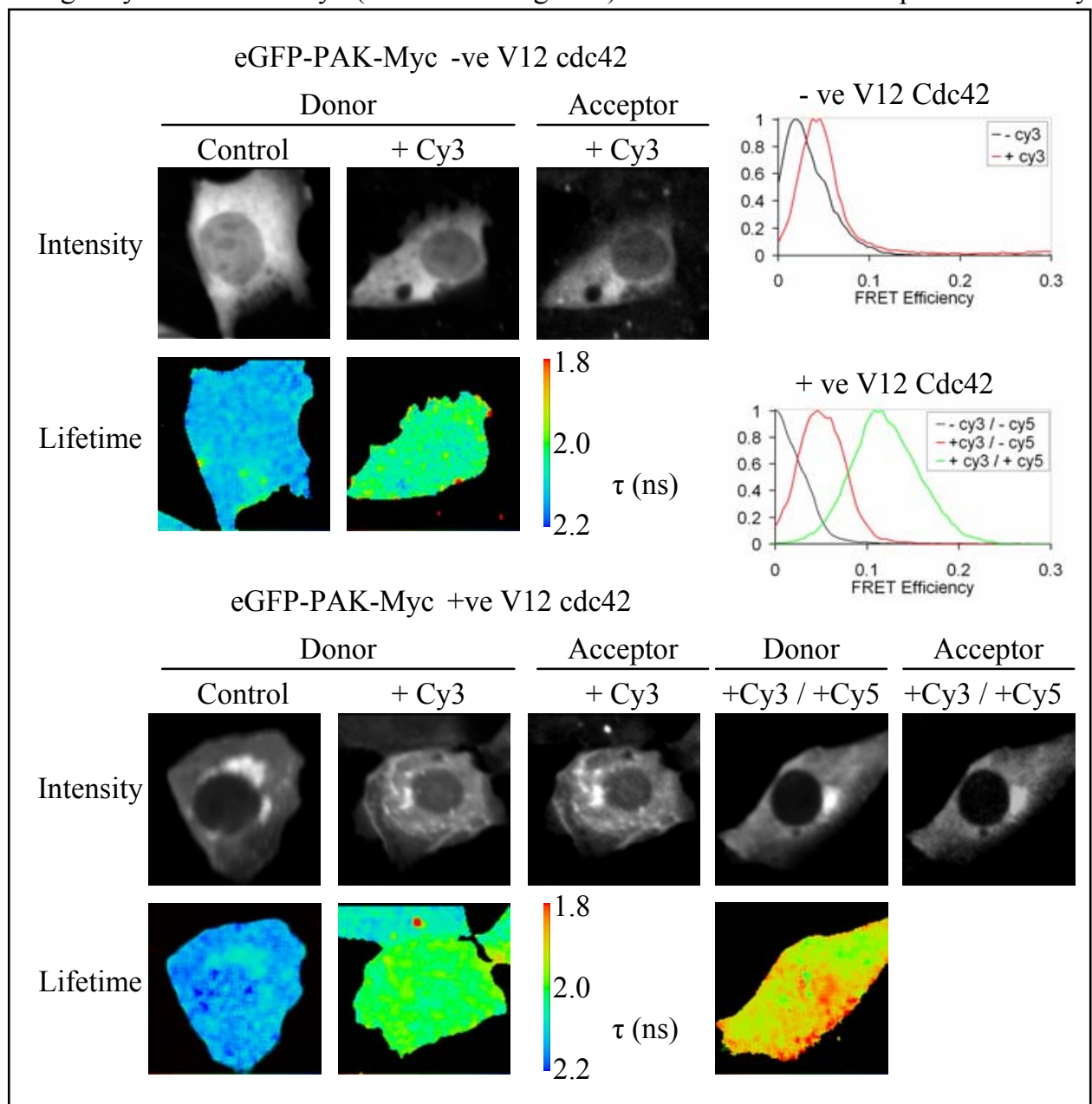

Figure 5; MP-FLIM data for the FRET cascade biosensor for PAK1 when cotransfected with the an active mutant of the Rho protein family member cdc42 (V12 cdc42).

FRET efficiency as measured for eGFP. Such an increase suggests that the presence of cy5, in some manner, affects the transfer between eGFP and cy3 or that transfer can occur directly between eGFP and cy5. Given the decrease in Förster radius with cy5 $(4 \mathrm{~nm})$ as opposed to cy3 $(6 \mathrm{~nm})$, direct transfer seems unlikely due to the physical proximity of the complex and the FRET efficiency observed in the presence of cy3 alone which should dominate the transfer process (due to the close proximity of the $\mathrm{C}$ and $\mathrm{N}$ terminus for the dimer). Addition of an unstained anti-Ha IgG or anti-HAIgG-cy5 alone had no effect on the measured lifetime compared to the controls. However, use of a cy5 labelled secondary antibody to the myc-cy3 IgG was found to significantly reduce the lifetime of eGFP as for the HA-IgG. One possible explanation is that the acceptor is rapidly bleached when cy 3 alone is present and that cy5 effectively protects 
cy3. Such phenomena are well known and have been exploited to measure FRET efficiency in steady state imaging ${ }^{22}$. In addition, the myc epitope may be masked to the antibody in the dimer configuration, which would lead to a less than optimum ratio of donors to acceptors (i.e. the target is not saturated with acceptors). This could lead to a bottleneck in the system (saturation), where multiple eGFP donors are in the vicinity of an acceptor. In this case, rapid saturation of the acceptors would manifest as a reduction in expected FRET efficiency. The mechanism by which the increase in FRET efficiency occurs has yet to be adequately explained and requires further exploration.

\section{CONCLUSIONS}

In conclusion, we have presented data for a new FRET pair (eGFP - mRFP) and discussed the relative merits of this approach in comparison with the excepted methodologies for quantitative FRET imaging of intra- and intermolecular protein interactions using multiphoton fluorescence lifetime imaging. We tentatively suggest that this methodology may prove superior to the well-established CFP-YFP cameleon, both in vivo and for fixed cell imaging.

We have also presented a new biosensor allowing simultaneous measurement of both conformational change and intramolecular binding. However, we have yet to propose a satisfactory model for the contrast observed in our experiments. Our future studies for this, so called, FRET cascade biosensor will aim to elucidate the mechanisms for enhanced FRET between primary donor-acceptor pairs, in the presence of a secondary acceptor.

\section{ACKNOWLEDGEMENTS}

We gratefully acknowledge the financial support of Cancer Research UK under Programme Grant C133/A1812. We would like to acknowledge the work of Dr J. Gilbey in development of analysis routines. We gratefully acknowledge the kind gift of mRFP1 from Prof. Roger Tsien (University of California, San Diego).

\section{REFERENCES}

1. Förster Th (1948). Zwischenmolekulare Energiewanderung und fluoreszenz. Annalen der Physik 6(2), pp55-75.

2. Ng T, Squire A, Hansra G, Bornancin F, Prevostel C, Hanby A, Harris W, Barnes D, Schmidt S, Mellor H, Bastiaens PIH and Parker PJ (1999). Imaging PKC alpha activation in cells. Science, 283, pp2085-2089.

3. Wouters FS, Verveer PJ and Bastiaens PIH (2001). Imaging Biochemistry inside cells. Trends Cell Biol. 11(5), pp203-211.

4. Becker W, Benndorf K, Bergmann A, Biskup C, König K, Tirplapur U and Zimmer T (2001). FRET measurements by TCSPC laser scanning microscopy. Proc. SPIE 4431, pp414-419.

5. Ameer-Beg SM, Barber PR, Hodgkiss RJ, Locke RJ, Newman RG, Tozer GM, Vojnovic B and Wilson J (2002). Application of multiphoton steady-state and lifetime imaging to mapping of tumour vascular architecture in vivo. Proc. SPIE 4620, pp. 85-95.

6. Habenicht A, Hjelm J, Mukhtar E, Bergstrom F, and Johansson LB-A (2002). Two-photon excitation and timeresolved fluorescence: I. The proper response function for analyzing single-photon counting experiments. Chem. Phys. Lett. 354, pp367-375.

7. Barber PR, Vojnovic B, Atkin G, Daley FM, Everett SA, Wilson GD (2003). Applications of cost-effective spectral imaging microscopy in cancer research. J. Phys. D: Applied Physics (in press).

8. Harpur AG and Bastiaens PIH Chapter 18: Protocol 5, probing protein interactions using eGFP and fluorescence resonance energy transfer,. Molecular Cloning: A Laboratory Manual (3rd edition), Cold Spring Harbor Laboratory Press, ISBN: 0879695773(Editors; Joseph Sambrook, David W. Russell, Joe Sambrook).

9. Rolinski OJ, Birch DJS, McCartney LJ and Pickup JC (2000). A method of determining donor-acceptor distribution functions in Förster resonance energy transfer. Chem. Phys. Lett. 324, pp95-100.

10. Lakowicz JR, Principles of fluorescence spectroscopy. Kluwer Academic/Plenum Publishers, ISBN 0-306-46093-9.

11. Verveer PJ, Squire A, Bastiaens PIH (2001). Global analysis of fluorescence lifetime imaging data. Biophys. J. 78, pp2127-2137.

12. Elangovan M, Day RN, Periasamy A (2002). Nanosecond fluorescence resonance energy transfer-fluorescence lifetime imaging microscopy to localize the protein interactions in a single living cell. J. Microscopy 205, pp3-14.

13. Campbell RE, Tour O, Palmer AE, Steinbach PA, Baird GS, Zacharias DA and Tsien RY (2002). A monomeric red fluorescent protein. Proc. Natl. Acad. Sci. 99(12), pp7877-7882. 
14. Tramier M, Gautier I, Piolot T, Ravalet S, Kemnitz K, Coppey J, Durieux C, Mignotte V and Coppey-Moisan M (2002). Picosecond-Hetero-FRET Microscopy to Probe Protein-Protein Interactions in Live Cells. Biophys J, 83, pp 3570-7.

15. Hanninen PE, Lehhtela L and Hell SW (1996). Two-and multiphoton excitation of conjugate-dyes using a continuous wave laser. Opt. Commun., 130, pp 29-33.

16. Schönle A, Hanninen PE, and Hell SW (1999). Non-linear fluorescence through intermolecular energy transfer and resolution increase in fluorescence microscopy. Ann. Phys. (Leipzig) 8(2), pp 115-133.

17. Miyawaki A, Griesbeck O, Heim R, and Tsien RY (1999). Dynamic and quantitative $\mathrm{Ca}^{2+}$ measurements using improved cameleons. Proc. Natl. Acad. Sci. 96, pp2135-2140.

18. Frost JA, Steen H, Shapiro P, Lewis T, Ahn N, Shaw PE, Cobb MH (1997). Cross-cascade activation of ERKs and ternary complex factors by Rho family proteins. EMBO 16(21), pp6426-6438.

19. Erickson JW and Cerione RA (2001). Multiple roles for Cdc42 in cell regulation. Current Opinion in Cell Biology 12, pp153-157.

20. Buchwald G, Hostinova E, Rudolphe MG, Kraemer A, Sickmann A, Meyer HE, Scheffzek K and Wittinghoffer A (2001). Conformational switch and role of phosphorylation in PAK activation. Molecular Cell Biology 21(15), pp5179-5189.

21. Parrini MC, Lei M, Harrison SC and Mayer BJ (2002). PAK1 kinase homodimers are autoinhibited in trans and dissociated upon activation by Cdc42 and Rac1. Molecular Cell 9, pp73-83.

22. Jovin TM and Arndt-Jovin DJ (1989). Luminescence digital imaging microscopy. Annu. Rev. Biophys. Chem. 18, 271-308. 